\title{
Effects of Dendropanax morbifera Léveille extracts on cadmium and mercury secretion as well as oxidative capacity: A randomized, double-blind, placebo-controlled trial
}

\author{
JAE SAM SEO ${ }^{1}$, DAE YOUNG YOO ${ }^{2}$, HYO YOUNG JUNG ${ }^{2}$, DONG-WOO KIM ${ }^{3}$, IN KOO HWANG ${ }^{2}$, \\ JONG YOUNG LEE ${ }^{4}$ and SEUNG MYUNG MOON ${ }^{1}$
}

\author{
${ }^{1}$ Department of Neurosurgery, Dongtan Sacred Heart Hospital, College of Medicine, Hallym University, Hwaseong 445-170; \\ ${ }^{2}$ Department of Anatomy and Cell Biology, College of Veterinary Medicine, and Research Institute for Veterinary Science, \\ Seoul National University, Seoul 151-742; ${ }^{3}$ Central Research Center, Egreen Co., Ltd., Seongnam 463-862; \\ ${ }^{4}$ Department of Neurosurgery, Kangdong Sacred Heart Hospital, College of Medicine, \\ Hallym University, Seoul 134-701, Republic of Korea
}

Received November 5, 2015; Accepted February 22, 2016

DOI: $10.3892 /$ br.2016.623

\begin{abstract}
In this randomized, double-blind, placebo controlled clinical trial, the effects of Dendropanax morbifera (D. morbifera) Léveille on heavy metal (cadmium and mercury) excretion as well as on lipid peroxidation and $\mathrm{Cu}, \mathrm{Zn}$-superoxide dismutase (SOD1) activity were investigated. For this study, tablets containing placebo or $300 \mathrm{mg}$ of the leaf extract from $D$. morbifera Léveille were used. A total of 60 eligible healthy subjects were enrolled in this randomized, double-blind, placebo-controlled study. The differences in cadmium, mercury, and malondialdehyde (MDA) levels and SOD1 activity were measured in the serum 60 days after treatment with placebo or D. morbifera Léveille extracts. No significant differences between baseline characteristics and biochemical values were identified in subjects in the placebo and D. morbifera Léveille groups. Serum levels of cadmium, mercury and MDA decreased following consumption of D. morbifera Léveille extracts; however, no significant differences were identified. In addition, female, but not male, subjects who consumed $D$. morbifera Léveille extracts showed a significant increase in SOD1 activity. This result suggests that chronic consumption of $D$. morbifera Léveille extract can help to facilitate excretion of cadmium and mercury from serum and increase the antioxidant capacity in humans.
\end{abstract}

Correspondence to: Professor Seung Myung Moon, Department of Neurosurgery, Dongtan Sacred Heart Hospital, College of Medicine, Hallym University, 40 Seoku-dong, Hwaseong 445-170, Republic of Korea

E-mail:nsmsm@chol.com

Key words: Dendropanax morbifera Léveille extract, cadmium, mercury, lipid peroxidation, $\mathrm{Cu}, \mathrm{Zn}$-superoxide dismutase

\section{Introduction}

Heavy metal intoxication and/or oxidative stress are implicated in numerous chronic diseases, including metabolic and neurological disorders. Environmental contamination by heavy metals such as cadmium $(\mathrm{Cd})$ and mercury $(\mathrm{Hg})$ is a serious international concern as these heavy metals can enter the food chain, accumulate in fishes and animals, and thus, endanger human health (1-5). According to the World Health Organization, the limit of tolerable intake for $\mathrm{Cd}$, methylmercury and inorganic $\mathrm{Hg}$ in an adult human is 7, 1.6 and $4 \mu \mathrm{g} / \mathrm{kg}$ of body weight per week, respectively (6).

One of the possible mechanisms underlying the effects of $\mathrm{Cd}$ and $\mathrm{Hg}$ is oxidative cytotoxicity. $\mathrm{Cd}$ and $\mathrm{Hg}$ interaction with the carboxyl and sulfhydryl group of proteins results in free radical generation (7-10). In addition, $\mathrm{Cd}$ and $\mathrm{Hg}$ can deplete antioxidant levels, resulting in an imbalance between oxidant and antioxidant ability. An increase in reactive oxygen species levels results in oxidation of polyunsaturated fatty acids, causing neuronal damage due to high acid content in the nervous system. In addition, our previous study demonstrated that $\mathrm{Cd}$ administration significantly increased lipid peroxidation and reduced levels of antioxidant enzymes such as $\mathrm{Cu}, \mathrm{Zn}$-superoxide dismutase (SOD1), catalase and glutathione peroxidase (11).

Dendropanax morbifera Léveille (DML), which belongs to the family Araliaceae, is an endemic species found in the southwestern region of South Korea (12). Recently, studies have investigated the effects of DML on various conditions, including their antioxidant and antidotal capacities $(11,13)$. For example, a methanol extract of the debarked stem of DML contains abundant phenolic and flavonoid compounds, which show strong antioxidant activities (13). In addition, our previous study demonstrated that DML extracts facilitate Cd excretion and suppress Cd-induced lipid peroxidation in hippocampal homogenates (11). However, to the best of our knowledge, no clinical study has examined the possible health benefits of a DML extract on heavy metal and antioxidant levels in human subjects. 
The aim of the present randomized, double-blind, placebo-controlled cross-over study was to examine the effects of repeated application of DML extract on the antioxidant status and oxidative damage in healthy adults.

\section{Subjects and methods}

Preparation of the test product from the leaf extract of $D M L$. The DML extract used was manufactured by a good manufacturing practices-certified company (Natural F\&P Corp., Cheonwon, Korea). Fresh leaves from DML were obtained from Hambakjae Biopharm Co., Ltd. (Jeju, Korea). The plant was authenticated by two practitioners of traditional Asian medicine. Leaves from the plant samples $(15 \mathrm{~kg})$ were chopped, blended and soaked in 2 liters of $80 \%$ ethanol, and refluxed three times at $20^{\circ} \mathrm{C}$ for $2 \mathrm{~h}$. The insoluble materials were removed by centrifugation at $10,000 \mathrm{x} \mathrm{g}$ for $30 \mathrm{~min}$, and the resulting supernatant was concentrated and freeze-dried to obtain a powder. For the test product preparation, DML, stearin magnesium and silicon dioxide were mixed. For the placebo, microcrystalline cellulose 102 was mixed with lactose powder, and dextrin was added to obtain a tablet form. Food colors (red, yellow and blue) were also added to produce the same color as the test product (Table I). The raw materials used in the test product and placebo were placed in a mixer and blended sufficiently to ensure homogeneity; subsequently, these were made into tablet at $300 \mathrm{mg} / \mathrm{tablet}$.

Stability testing for the test product and placebo. The test product and placebo were assessed for stability; moisture content (\%); coliform group; lead (mg/kg), arsenic ( $\mathrm{mg} / \mathrm{kg}$ ) and sodium (mg/100 g) levels; and disintegration by outsourcing to 'Korea Health Supplements Association' (Seongnam, South Korea). All test results were acceptable as per the requirements set by the Food Sanitation Act. No preservative was added to the test product.

Study approval and sample target group selection. In accordance with the Functional Foods for Health Act (http://www. mfds.go.kr/files/upload/eng/FOOD_SANITATION_ACT.pdf), all issues associated with human trials were approved by the Institutional Review Board for Human Trials of Hallym University (Hwaseong, Korea) (IRB approval no. 2014-074); the study was conducted in agreement with the Declaration of Helsinki and performed in accordance with International Conference on Harmonization guidelines. In addition, the study was retrospectively registered at the WHO Primary Registries in Republic of Korea (Clinical Research Information Service) (registration no. KCT0001472, May 8, 2015).

Subjects were male and female volunteers aged 23-65 years $(n=61)$. The subjects were informed of the details of the trial, and only those who fully understood the purpose of trial participation and gave consent for participation were selected as subjects. All the subjects were considered suitable for the trial as they had normal liver functions, as assessed by measuring glutamate-oxaloacetate transaminase (AST) and glutamate-pyruvate transaminase (ALT) levels in the blood. Exclusion criteria consisted of intestinal absorption abnormalities and liver functions. Subjects with ALT or AST $>60 \mathrm{U} / \mathrm{T}$ were also excluded. One subject was excluded due
Table I. Composition of Dendropanax morbifera Léveille (DML) extracts and placebo tablets.

\begin{tabular}{|c|c|c|}
\hline Composition & DML & Placebo (\%) \\
\hline Dendropanax extracts & 98.000 & \\
\hline Cellulose 102 & & 59.852 \\
\hline Lactose powder & & 39.600 \\
\hline Stearin magnesium & 1.000 & \\
\hline Dextrin & & 0.400 \\
\hline Silicon dioxide & 1.000 & \\
\hline Food color (red/yellow/blue) & & 0.148 \\
\hline Total & 100.000 & 100.000 \\
\hline
\end{tabular}

to high levels of ALT and AST. The others (n=60) (Fig. 1) were randomly assigned to treatment groups, minimizing any intentional elements of group assignment, in a double-blind procedure to ensure that the subjects did not know which group they were assigned to (test group or comparative control group) (Fig. 1). The sample size of a minimum 9 per treatment group would provide $\geq 95 \%$ power to detect a difference of $\mathrm{Cd}$ values in the serum based on the previous animal study (11).

Analytical procedures. For heavy metal, lipid peroxidation and antioxidant analysis, the assay was performed prior and subsequent to administration of the test product and the placebo (Fig. 2). All the subjects were instructed to ingest the tablets after breakfast over a 60-day period. The placebo group was comparable in all characteristics to the DML group. Food and water intake was not limited.

Measurement of $\mathrm{Cd}, \mathrm{Hg}$ and malondialdehyde (MDA) levels and SODI activity in the serum. By vein puncture with a syringe, blood samples were collected in tubes containing ethylenediaminetetraacetic acid. The tubes were immediately centrifuged at $4^{\circ} \mathrm{C}$ and $500 \mathrm{x}$ g for $10 \mathrm{~min}$ to obtain serum and plasma for measuring $\mathrm{Cd}, \mathrm{Hg}$ and MDA levels and SOD1 activity. These parameters were measured using routine automated methods. Adverse experiences were rated by the investigators for intensity and their association to the DML extract. Efficacy and safety measurements were conducted at a Hallym University Dongtan Sacred Heart Hospital (Dongtan, South Korea) laboratory and at a Seoul National University College of Veterinary Medicine (Seoul, South Korea) by technicians or graduate students blinded to the placebo and treatment groups.

Statistical analysis. The measured $\mathrm{Hg}$ and $\mathrm{Cd}$ levels were calculated into excretion ratio (delta\% value), as shown in Fig. 2. The MDA levels and SOD1 activities 60 days after placebo or DML consumption are shown. All the data are expressed as mean \pm standard error of the mean. To determine the effects of the DML tablet, differences between the means of the two groups were statistically analyzed using two-way analysis of variance with repeated measures and Bonferroni's post hoc test using GraphPad Prism 5.01 (GraphPad Software, La Jolla, CA, USA) software. 


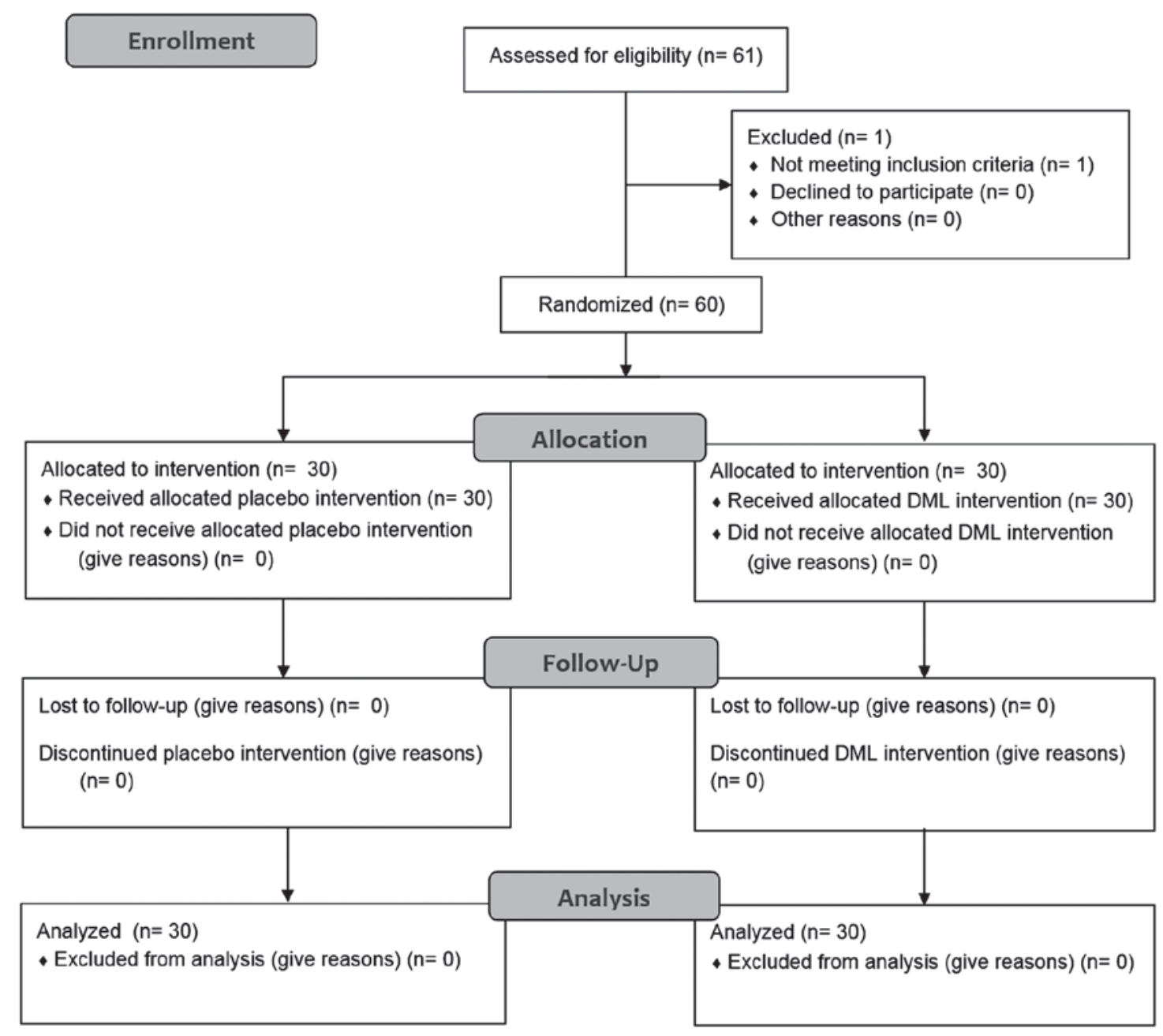

Figure 1. Consort flow diagram of the patients enrolled, allocated, followed up and analyzed during the study.

\section{Results}

Patient characteristics. The baseline characteristics and biochemical values of the subjects did not significantly differ between the placebo and DML groups (data not shown). In addition, no significant differences between male and female participants were identified.

$\mathrm{Cd}$ and $\mathrm{Hg}$ excretion. Serum Cd levels were increased in the male and female subjects in the placebo group and decreased in the DML group; however, no significant difference between the two groups was identified ( $\mathrm{P}=0.3008$ ) (Fig. 3). Serum $\mathrm{Hg}$ levels decreased in all the groups, but serum levels were more prominently decreased in the DML-male and DML-female groups compared to the placebo-male and placebo-female groups, respectively $(\mathrm{P}=0.1336$ and $\mathrm{P}=0.1937$, respectively) (Fig. 3).

Oxidative stress biomarkers. Serum MDA levels were increased in the DML-male group compared to the placebo-male group $(\mathrm{P}=0.3125)$, while these were decreased in the DML-female group compared to the placebo-female group ( $\mathrm{P}=0.0842)$ (Fig.4); however, no significant differences were identified.

Serum SOD1 activity was increased in DML-treated groups, but the SOD1 activity was significantly increased in the DML-female group compared to the placebo-female group $(\mathrm{P}=0.02031)$. However, no significant difference in SOD1 activity was observed between the placebo-male and DML-male group ( $\mathrm{P}=0.2031)$ (Fig. 4).

\section{Discussion}

Several lines of evidence demonstrate that DML and its components reduce reactive oxygen species generation and have antioxidant effects $(13,14)$. In addition, our previous study demonstrated that DML facilitates Cd excretion and exerts antioxidant effects against $\mathrm{Cd}$-induced oxidative damage (11). The present study attempted to use DML to facilitate $\mathrm{Cd}$ and $\mathrm{Hg}$ excretion in humans and to increase the antioxidant capacity in the serum, as $\mathrm{Cd}$ and $\mathrm{Hg}$ have been shown to inhibit antioxidant enzymes (15-17).

No significant decrease in serum $\mathrm{Cd}$ or $\mathrm{Hg}$ levels was observed. However, the consumption of the DML tablet for 2 months facilitated excretion of $\mathrm{Cd}$ to $4.6 \%$ in male subjects and $\mathrm{Hg}$ to 23.0 and $17.3 \%$ in male and female subjects, respectively. This result suggests that consumption of DML tablet facilitates $\mathrm{Cd}$ and $\mathrm{Hg}$ excretion from the serum. The facilitated excretion rate is relatively low for $\mathrm{Cd}$; however, the facilitation is extremely important as cadmium is tightly bound to metallothionein, which is almost completely reabsorbed in the 
$\mathbf{0 ~ d}$

$60 \mathrm{~d}$

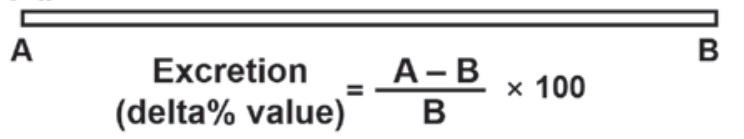

\begin{tabular}{|l|c|c|c|c|}
\hline & \multicolumn{2}{|c|}{ Placebo } & \multicolumn{2}{c|}{ DML } \\
\hline Gender & Male & Female & Male & Female \\
\hline Number & 9 & 21 & 16 & 14 \\
\hline Age & $29.00 \pm 11.95$ & $27.14 \pm 5.83$ & $29.11 \pm 7.82$ & $32.57 \pm 10.09$ \\
\hline
\end{tabular}

Figure 2. Experimental design and baseline characteristics (gender, number and age) of the subjects in the present study.

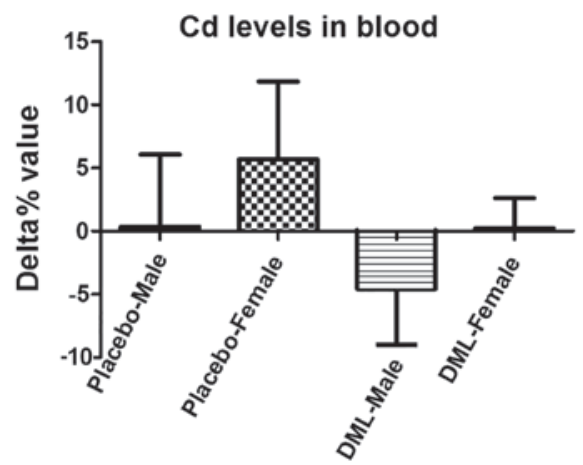

$\mathrm{Hg}$ levels in blood

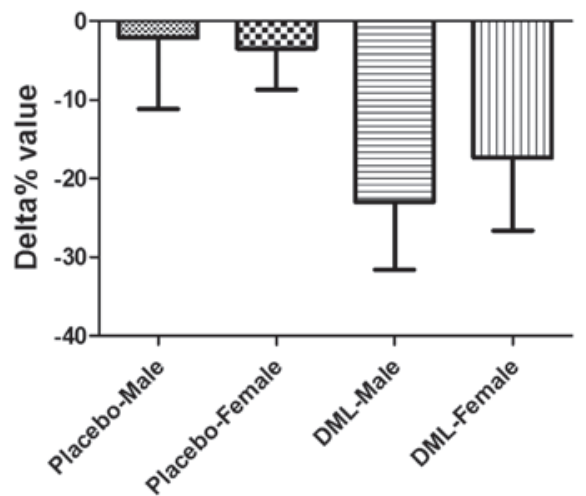

Figure 3. Effects of Dendropanax morbifera Léveille (DML) extract on cadmium $(\mathrm{Cd})$ and mercury $(\mathrm{Hg})$ concentration in the blood of subjects who consumed placebo and DML. The data represent the mean \pm standard error of the mean.

renal tubules and accumulates in the body over the lifetime with a biological half-life of $\leq 38$ years (6).

Heavy metals contribute to oxidative processes $(18,19)$, and the levels of reactive oxygen species are important factors associated with the development of chronic disease $(20,21)$. In the present study, the levels of MDA, a well-known product of lipid peroxidation $(22,23)$, were measured in human subjects. MDA levels were decreased in the DML group compared to the placebo group. This result was consistent with our previous study showing that supplementation with the DML suppressed Cd-induced increase of MDA levels, as well as protein carbonylation due to modified proteins in the rat hippocampus (11). In the present study, the DML significantly increased SOD levels in the female treatment group compared to the female

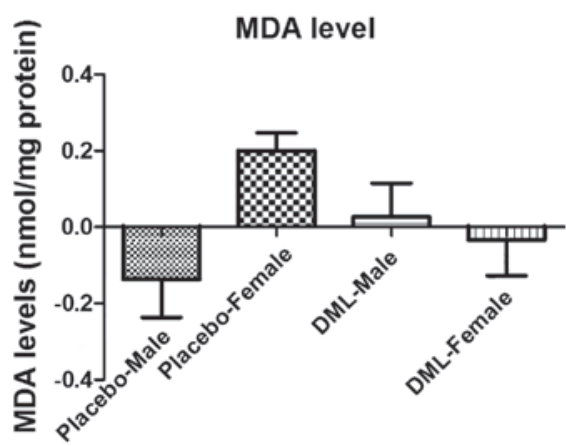

SOD activity

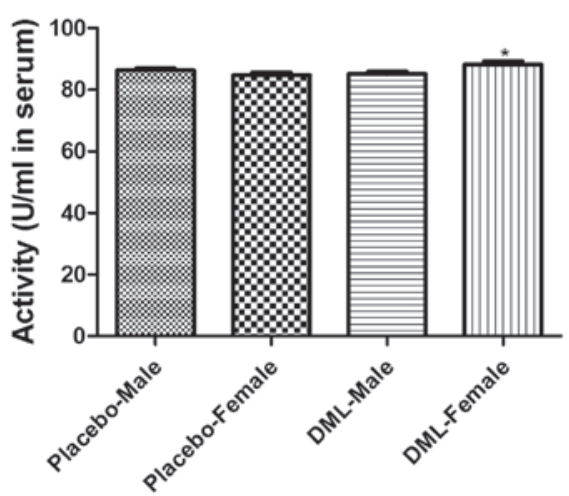

Figure 4. Malondialdehyde (MDA) levels and superoxide dismutase 1 (SOD1) activity in the serum of subjects who consumed placebo and Dendropanax morbifera Léveille (DML) extract. Significant difference between the placebo and DML groups ( $\mathrm{P}<0.05)$. The data represent means \pm standard error of the mean.

placebo group; this suggests that treatment with the extract for 2 months increases the antioxidant capacity in the blood. This is consistent with our previous study showing that administration of DML increased SOD1 activity in normal healthy rats (11).

In conclusion, administration of DML has positive effects on excretion of $\mathrm{Cd}$ and $\mathrm{Hg}$ from the blood and increases antioxidant capacity by decreasing MDA levels and increasing SOD activity in the serum.

\section{Acknowledgements}

The present study was supported by the High Value-added Food Technology Development Program, Ministry for 
Agriculture, Food and Rural Affairs, Republic of Korea (grant no. 112106-022-HD020).

\section{References}

1. Kim NS and Lee BK: National estimates of blood lead, cadmium, and mercury levels in the Korean general adult population. Int Arch Occup Environ Health 84: 53-63, 2011.

2. Vieira C, Morais S, Ramos S, Delerue-Matos C and Oliveira MB Mercury, cadmium, lead and arsenic levels in three pelagic fish species from the Atlantic Ocean: Intra- and inter-specific variability and human health risks for consumption. Food Chem Toxicol 49: 923-932, 2011.

3. Jain RB: Effect of pregnancy on the levels of blood cadmium, lead, and mercury for females aged 17-39 years old: Data from National Health and Nutrition Examination Survey 2003-2010. J Toxicol Environ Health A 76: 58-69, 2013.

4. Tyrrell J, Melzer D, Henley W, Galloway TS and Osborne NJ: Associations between socioeconomic status and environmental toxicant concentrations in adults in the USA: NHANES 2001-2010. Environ Int 59: 328-335, 2013.

5. Storelli MM, Barone G, Cuttone G, Giungato D and Garofalo R: Occurrence of toxic metals $(\mathrm{Hg}, \mathrm{Cd}$ and $\mathrm{Pb})$ in fresh and canned tuna: Public health implications. Food Chem Toxicol 48: 3167-3170, 2010.

6. Agency for Toxic Substances and Disease Registry: Toxicological profile for cadmium. US Department of Health and Human Services. NTIS Report, Atlanta, GA, 1999. No. PB/89/194476/AS

7. Ates I, Suzen HS, Aydin A and Karakaya A: The oxidative DNA base damage in testes of rats after intraperitoneal cadmium injection. Biometals 17: 371-377, 2004.

8. Shanker G, Aschner JL, Syversen T and Aschner M: Free radical formation in cerebral cortical astrocytes in culture induced by methylmercury. Brain Res Mol Brain Res 128: 48-57, 2004.

9. Gałazyn-Sidorczuk M, Brzóska MM, Jurczuk M and Moniuszko-Jakoniuk J: Oxidative damage to proteins and DNA in rats exposed to cadmium and/or ethanol. Chem Biol Interact 180: 31-38, 2009.

10. Gonçalves JF, Fiorenza AM, Spanevello RM, Mazzanti CM, Bochi GV, Antes FG, Stefanello N, Rubin MA, Dressler VL, Morsch VM, et al: N-acetylcysteine prevents memory deficits, the decrease in acetylcholinesterase activity and oxidative stress in rats exposed to cadmium. Chem Biol Interact 186: 53-60, 2010.

11. Kim W, Kim DW, Yoo DY, Jung HY, Nam SM, Kim JW, Hong SM, Kim DW, Choi JH, Moon SM, et al: Dendropanax morbifera Léveille extract facilitates cadmium excretion and prevents oxidative damage in the hippocampus by increasing antioxidant levels in cadmium-exposed rats. BMC Complement Altern Med 14: 428, 2014.
12. Han S, Jung Y, Ko M, Oh Y, Koh S, Kim M and Oh M: Phylogenetic relationships of the Dendropanax morbifera and D-trifidus based on PCR-RAPD. Korean J Genet 20: 173-181, 1998.

13. Hyun TK, Kim MO, Lee H, Kim Y, Kim E and Kim JS: Evaluation of anti-oxidant and anti-cancer properties of Dendropanax morbifera Léveille. Food Chem 141: 1947-1955, 2013.

14. Park SE, Sapkota K, Choi JH, Kim MK, Kim YH, Kim KM, Kim KJ, Oh HN, Kim SJ and Kim S: Rutin from Dendropanax morbifera Leveille protects human dopaminergic cells against rotenone induced cell injury through inhibiting JNK and p38 MAPK signaling. Neurochem Res 39: 707-718, 2014.

15. Amara S, Douki T, Garrel C, Favier A, Ben Rhouma K, Sakly M and Abdelmelek H: Effects of static magnetic field and cadmium on oxidative stress and DNA damage in rat cortex brain and hippocampus. Toxicol Ind Health 27: 99-106, 2011.

16. Agrawal S, Flora G, Bhatnagar P and Flora SJ: Comparative oxidative stress, metallothionein induction and organ toxicity following chronic exposure to arsenic, lead and mercury in rats. Cell Mol Biol (Noisy-le-grand) 60: 13-21, 2014.

17. Martinez CS, Escobar AG, Torres JG, Brum DS, Santos FW, Alonso MJ,Salaices M, Vassallo DV,PeçanhaFM,Leivas FG, et al: Chronic exposure to low doses of mercury impairs sperm quality and induces oxidative stress in rats. J Toxicol Environ Health A 77: 143-154, 2014.

18. Stohs SJ and Bagchi D: Oxidative mechanisms in the toxicity of metal ions. Free Radic Biol Med 18: 321-336, 1995.

19. Ercal N, Gurer-Orhan H and Aykin-Burns N: Toxic metals and oxidative stress part I: Mechanisms involved in metal-induced oxidative damage. Curr Top Med Chem 1: 529-539, 2001.

20. Basu S: F2-isoprostanes in human health and diseases: From molecular mechanisms to clinical implications. Antioxid Redox Signal 10: 1405-1434, 2008.

21. Galanis A, Karapetsas A and Sandaltzopoulos R: Metal-induced carcinogenesis, oxidative stress and hypoxia signalling. Mutat Res 674: 31-35, 2009.

22. Romero FJ, Bosch-Morell F, Romero MJ, Jareño EJ, Romero B, Marín N and Romá J: Lipid peroxidation products and antioxidants in human disease. Environ Health Perspect 106 (Suppl 5): 1229-1234, 1998.

23. Del Rio D, Stewart AJ and Pellegrini N: A review of recent studies on malondialdehyde as toxic molecule and biological marker of oxidative stress. Nutr Metab Cardiovasc Dis 15: 316-328, 2005. 